\title{
Generation Y und Arbeitgeberattraktivität des Mittelstands
}

\author{
Extrakt des Beitrags "Generation Y und der Mittelstand - Best \\ Practices für Mitarbeitergewinnung, -entwicklung und -bindung." \\ von Bianca Arndt, Matthias D. Mahlendorf und Martin Schomaker* \\ erschienen in: Schmalenbach IMPULSE, 2. Jg. 2022, Heft 2, \\ https://doi.org/ 10.54585/GTBL2517.
}

Die Zahl der Menschen im Erwerbsalter wird bis $2035 \mathrm{im}$ moderaten Szenario um 5,4 Mio. sinken. Angesichts der zu erwartenden Arbeitskräfteknappheit zählt es zu den wichtigsten Handlungsfeldern des Personalmanagements, sich mit der jungen Generation auseinanderzusetzen und stärker auf ihre Bedürfnisse einzugehen. Insbesondere kleinere, mittelständische Unternehmen müssen sich der Herausforderung stellen, im Wettstreit um die Talente als attraktiver Arbeitgeber wahrgenommen zu werden.

Die junge Generation hat deutlich höhere Erwartungen an die Arbeit und den Arbeitgeber, als dies bei den Vorgängergenerationen der Fall war. Mit dem Bewusstsein, dass talentierte und gut qualifizierte Arbeitskräfte rar sind, formuliert die Generation Y selbstbewusst meist hohe Ansprüche an potenzielle Arbeitgeber:

- Flexibilität und Work-Life-Balance

- Weiterentwicklungs- und Karrieremöglichkeiten

- Kooperativer Führungsstil und Feedback

- Teamorientierte und sinnstiftende Unternehmenskultur

- Leistungsbezogenheit, Gerechtigkeit und Angemessenheit der Vergütung

\footnotetext{
Bianca Arndt | Master Graduate in Finance

Dr. Matthias D. Mahlendorf | Professor of Managerial Accounting | Frankfurt School of Finance \& Management | m.mahlendorf@fs.de

Martin Schomaker | Privatier und Aufsichtsrat
} 
Zur Generation Y werden dabei üblicherweise die Personen gerechnet, die zwischen 1980 und ca. 2000 geboren wurden.

\section{Arbeitgeberattraktivität des Mittelstands}

Die spezifischen Stärken und Schwächen des Mittelstands als Arbeitgeber in diesem Kontext im Vergleich zum Großunternehmen lassen sich wie folgt zusammenfassen:

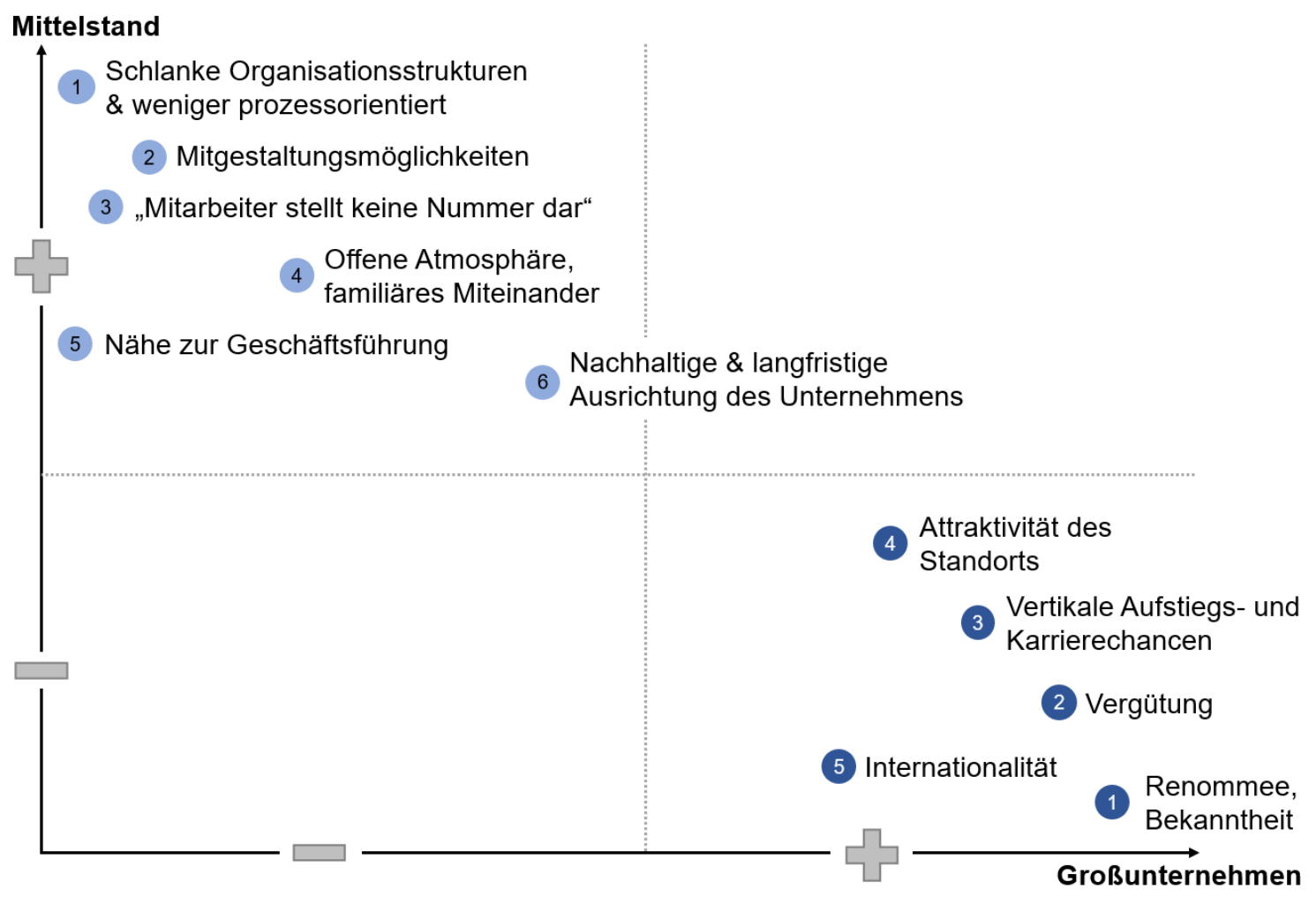

Abb. 1: Stärken-Schwächen-Profil des Mittelstandes

\section{Maßnahmen zur Steigerung der Arbeitgeberattraktivität}

Auf Basis einer umfangreichen Literaturrecherche, Experteninterviews und einer Round Table Diskussion im AK Wertorientierte Führung in mittelständischen Unternehmen der Schmalenbach-Gesellschaft für Betriebswirtschaft e.V. identifizieren die Autor:innen besonders erfolgversprechende Ansätze um sich von Großunternehmen abzuheben.

Im Bereich der Mitarbeitergewinnung spielt der persönliche Einsatz der Top-Führungskräfte - zum Beispiel über soziale Netzwerke aber auch bei klassischen Rekrutierungsveranstaltungen - eine zentrale Rolle. 


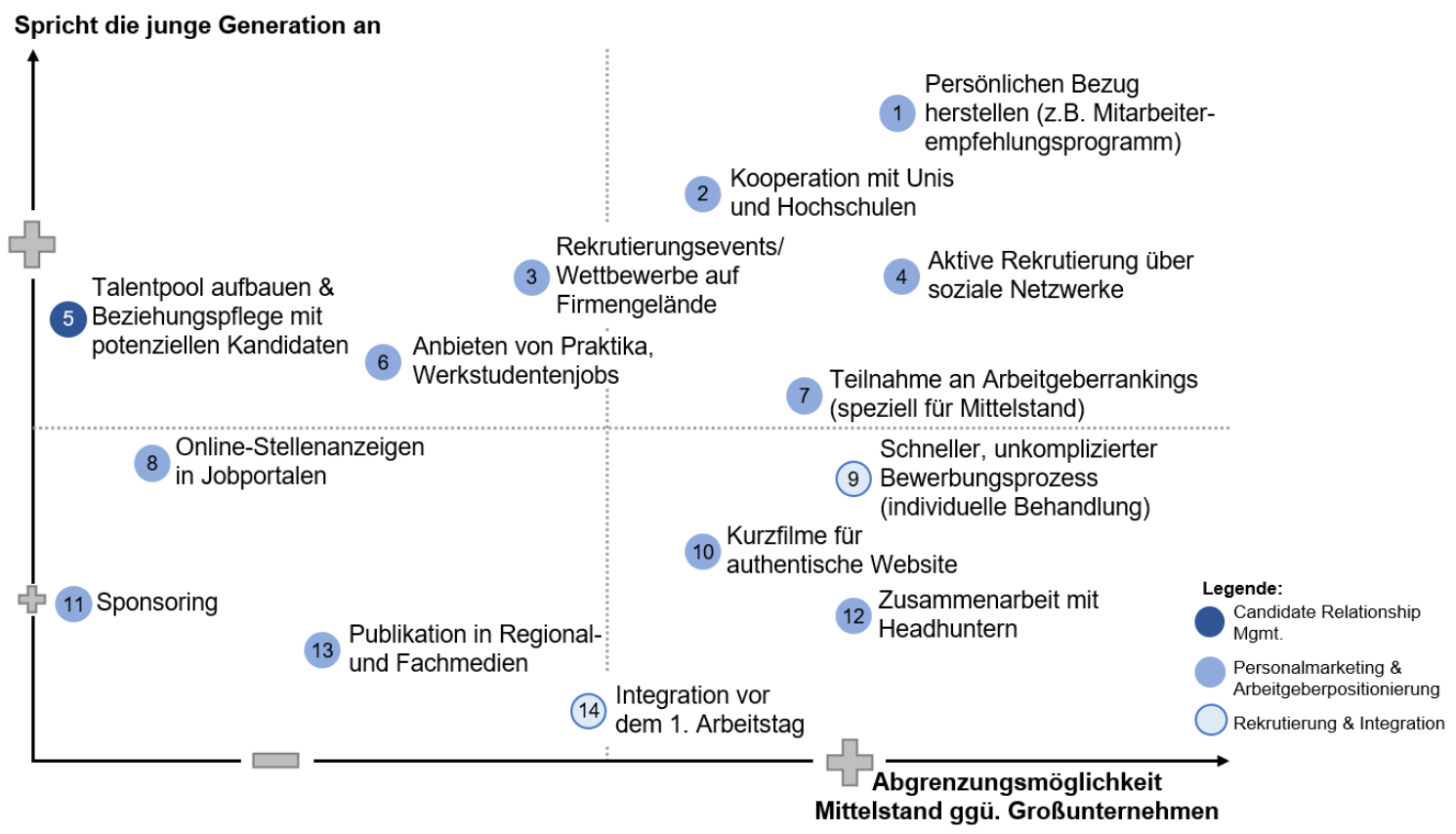

Abb. 2: Maßnahmen zur Verbesserung der Mitarbeitergewinnung

Hinsichtlich der Mitarbeiterentwicklung bietet die individualisierte Förderung durch die Übertragung von Projektverantwortung, gezielte Schulung sowie die intelligente Karriereplanung im Sinne einer „Kletterwand mit vielen Möglichkeiten“ Potential sich zu differenzieren. Neben einem Paten- oder Mentorenmodell können durch die aktuelle Verbesserung der mobilen Arbeitsmöglichkeiten Standort-Rotationen zur Horizonterweiterung auch im Mittelstand umgesetzt werden.

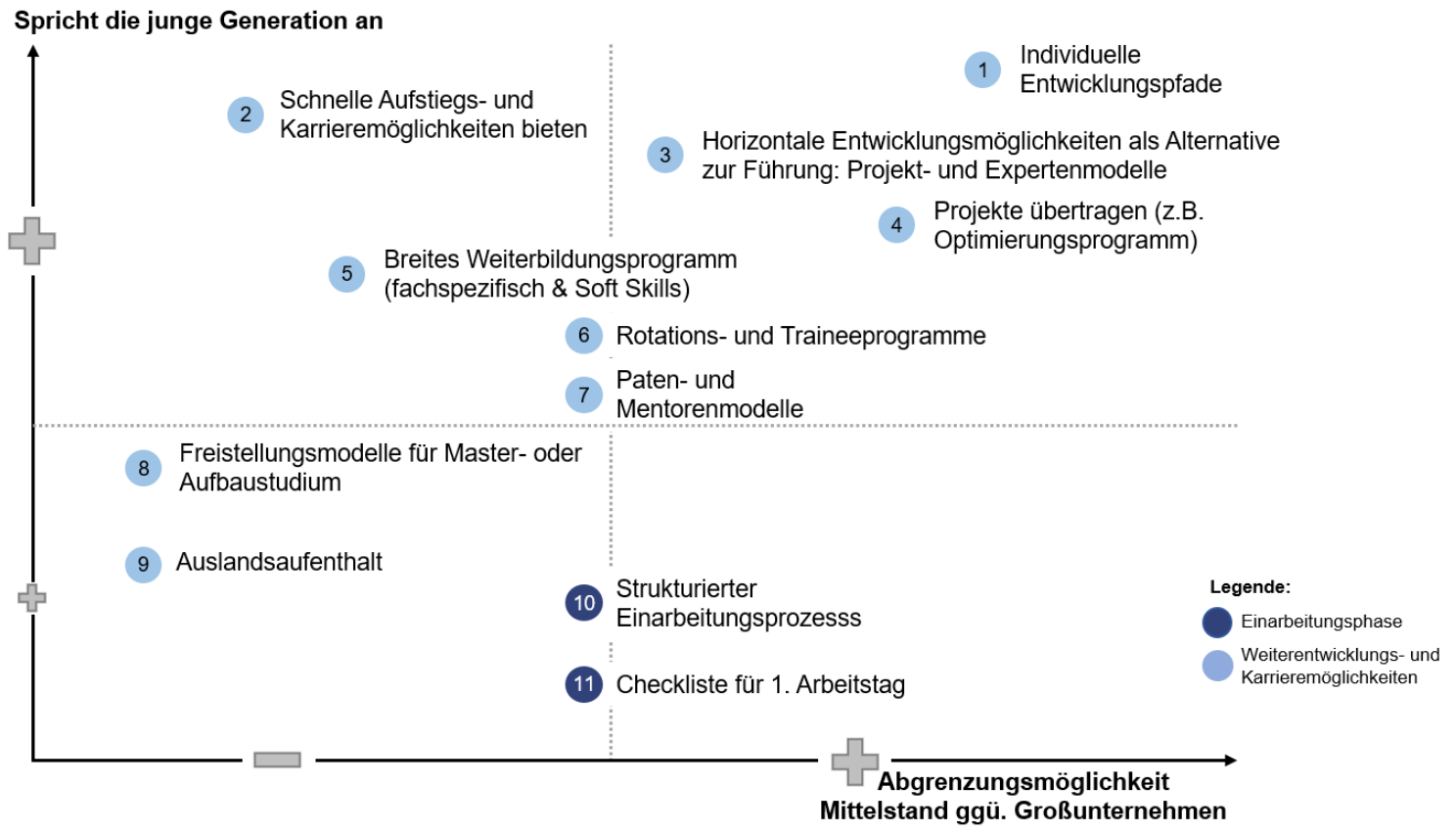

Abb. 3: Maßnahmen zur Verbesserung der Mitarbeiterentwicklung 
Was die Mitarbeiterbindung betrifft, ist es zum einen wichtig, schneller größere Entscheidungsfreiräume und Mitgestaltungsmöglichkeiten anzubieten als dies in Großunternehmen der Fall ist. Zum anderen ist auch die soziale Anerkennung und Wertschätzung - beispielsweise unterstützt durch moderne Tools wie bonusly - ein wichtiger Hebel. Eine innovative Unternehmenskultur mit zeitnahem Feedback und hoher Transparenz, wie sie zum Beispiel durch moderne Tools wie Objectives und Key Results (OKR) entsteht, spricht die Millennials an. Gekoppelt mit dem überschaubaren familiären Unternehmen, in dem mit Gemeinschaftsveranstaltungen der Zusammenhalt gepflegt wird, ist das ein vielversprechender Ansatz.

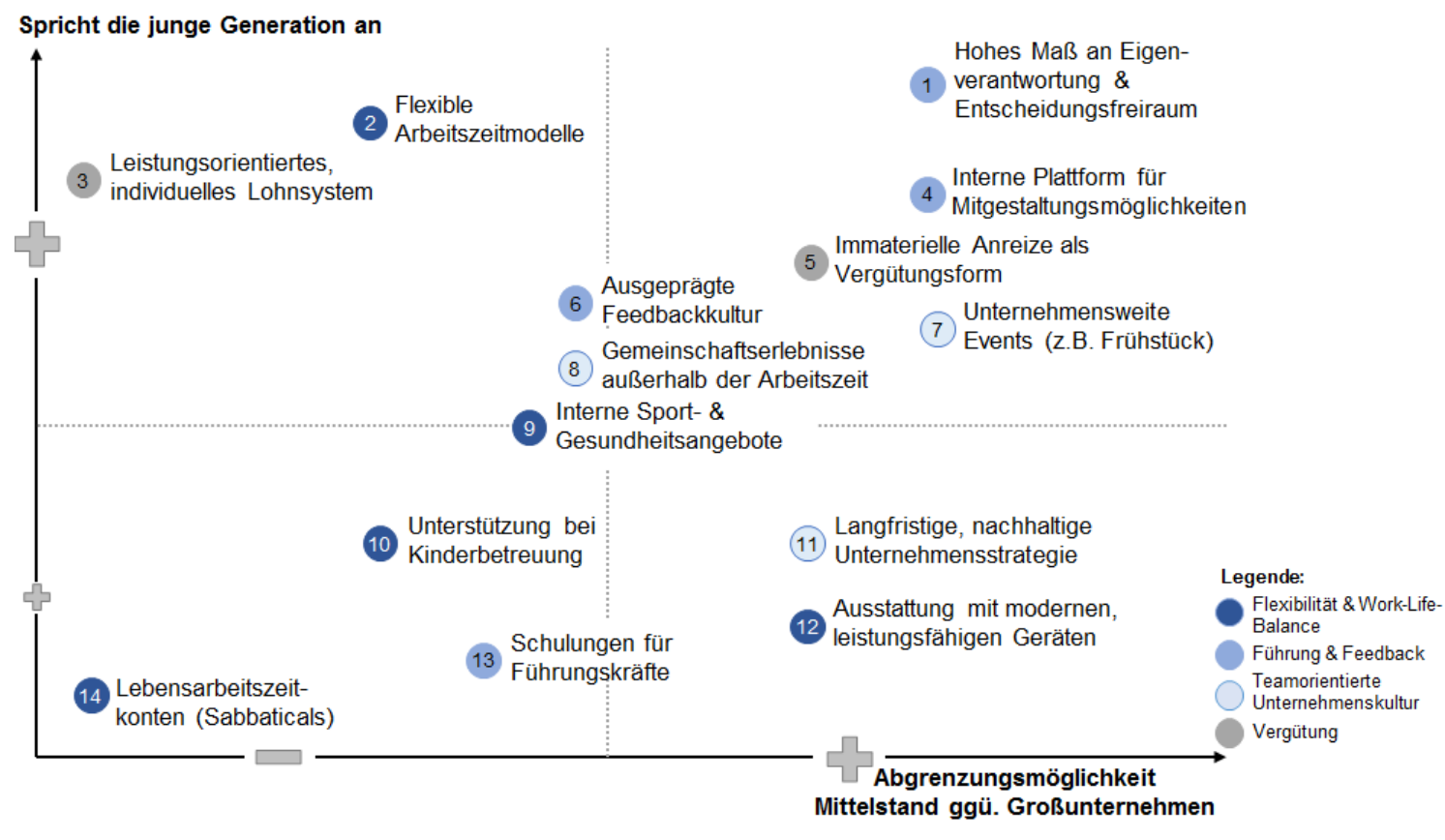

Abb. 4: Maßnahmen zur Verbesserung der Mitarbeiterbindung

Zusammenfassend stellen die Autor:innen fest: Legt man die die Stärken des Mittelstands mit den Werten der jungen Generation übereinander, ergibt sich eine Reihe von Chancen des Mittelstands, als attraktiver Arbeitgeber wahrgenommen zu werden. 